Asia Pac. J. Math. 2020 7:8

1210

ASIA PACIFIC ACADEMIC

\title{
INCOMPLETENESS IN THE BELL THEOREM INVOLVING ALL SETTINGS OF MEASURING APPARATUS
}

\section{KOJI NAGATA ${ }^{1, *}$, SANTANU KUMAR PATRO ${ }^{2}$, SHAHROKH HEIDARI ${ }^{3}$, GERMANO RESCONI ${ }^{4}$, DO NGOC DIEP $^{5,6}$, TADAO NAKAMURA $^{7}$, HAN GEURDES $^{8}$}

\author{
${ }^{1}$ Department of Physics, Korea Advanced Institute of Science and Technology, Daejeon 34141, Korea \\ ${ }^{2}$ Incessive Education \& Research (P) Ltd, Odisha-761055, India \\ ${ }^{3}$ Young Researchers and Elite Club, Kermanshah Branch, Islamic Azad University, Kermanshah, Iran \\ ${ }^{4}$ Department of Mathematics and Physics, Catholic University, Brescia, Italy \\ ${ }^{5}$ TIMAS, Thang Long University, Nghiem Xuan Yem road, Hoang Mai district, Hanoi, Vietnam \\ ${ }^{6}$ Institute of Mathematics, VAST, 18 Hoang Quoc Viet road, Cau Giay district, Hanoi, Vietnam \\ ${ }^{7}$ Department of Information and Computer Science, Keio University, 3-14-1 Hiyoshi, Kohoku-ku, Yokohama 223-8522, Japan \\ ${ }^{8}$ Geurdes Datascience, KvK 64522202, C vd Lijnstraat 164, 2593 NN, Den Haag Netherlands \\ *Corresponding author: ko_mi_na@yahoo.co.jp \\ Received Mar. 10, 2020
}

\begin{abstract}
Aвstract. Here, we consider the Bell experiment for a system described by multipartite states in the case where $n$ dichotomic observables are measured per site. If $n$ is two, we consider a two-setting Bell experiment. If $n$ is infinite, we consider an infinite-setting Bell experiment. Two-setting model is an explicit local realistic model for the values of a correlation function, given in a two-setting Bell experiment. Infinite-setting model is an explicit local realistic model for the values of a correlation function, given in an infinite-setting Bell experiment. In the non-contextual scenario, there is not the essential difference between infinite-setting model and two-setting model. And we cannot classify local realistic theories in this case. This says that we can construct infinite-setting model from two-setting model. Surprisingly we can discuss incompleteness in the Bell theorem by using non-contextual models. On the other hand, in the contextual scenario, there is the essential difference between infinite-setting model and two-setting model. This says that we must distinguish infinite-setting model from two-setting model. And we can classify local realistic theories in this case.
\end{abstract}

Mathematics Subject Classification: 81P15.

Key words and phrases: quantum non locality; quantum measurement theory; formalism.

\section{INTRODUCTION}

Non-locality in quantum physics means the possibility of distributing correlations that cannot be due to previously shared randomness, without signaling [1]. Some quantum predictions violate Bell inequalities [2], which form necessary conditions for local realistic theories for the results of measurements. Thus, some quantum predictions do not accept local realistic theories. Experimental efforts (Bell experiment) of a violation of local

DOI: $10.28924 / \mathrm{APJM} / 7-8$

(92020 Asia Pacific Journal of Mathematics 
realism can be seen in [3-5]. Other types of inequalities are given in [6,7]. Bell inequalities with settings other than spin polarizations can be seen in [8].

We consider the Bell experiment for a system described by multipartite states in the case where $n$-dichotomic observables are measured per site. If $n$ is two, we consider a two-setting Bell experiment. If $n$ is infinite, we consider an infinite-setting Bell experiment. Two-setting model is an explicit local realistic model for the values of a correlation function, given in a two-setting Bell experiment. Infinite-setting model is an explicit local realistic model for the values of a correlation function, given in an infinite-setting Bell experiment.

In the non-contextual scenario, there is not the essential difference between infinite-setting model and twosetting model. And we cannot classify local realistic theories in this case. This says that we can construct infinite-setting model from two-setting model. Surprisingly we can discuss incompleteness $[9,10]$ in the Bell theorem by using non-contextual models. On the other hand, in the contextual scenario, there is the essential difference between infinite-setting model and two-setting model. This says that we must distinguish infinitesetting model from two-setting model. And we can classify local realistic theories in this case [11,12].

\section{INCOMPLETENESS IN THE BELL THEOREM INVOLVING ALL SETTINGS OF MEASURING APPARATUS}

Assume that we have a set of $N$ spins $\frac{1}{2}$. Each of them is in a separate laboratory. As is well known the measurements (observables) for such spins are parameterized by a unit vector $\vec{n}_{j}, j=1,2, \ldots, N$ (its direction along which the spin component is measured). The results of measurements are \pm 1 (in $\hbar / 2$ unit). We can introduce the "Bell" correlation function, which is the average of the product of the local results:

$$
E\left(\vec{n}_{1}, \vec{n}_{2}, \ldots, \vec{n}_{N}\right)=\left\langle r_{1}\left(\vec{n}_{1}\right) r_{2}\left(\vec{n}_{2}\right) \cdots r_{N}\left(\vec{n}_{N}\right)\right\rangle_{\mathrm{avg}}
$$

where $r_{j}\left(\vec{n}_{j}\right)$ is the local result, \pm 1 , which is obtained if the measurement direction is set at $\vec{n}_{j}$.

If an experimental correlation function admits rotationally invariant tensor structure familiar from Newton's theory, we can introduce the following form:

$$
E\left(\vec{n}_{1}, \vec{n}_{2}, \ldots, \vec{n}_{N}\right)=\hat{T} \cdot\left(\vec{n}_{1} \otimes \vec{n}_{2} \otimes \cdots \otimes \vec{n}_{N}\right),
$$

where $\otimes$ denotes the tensor product, $\cdot$ denotes the scalar product in $\mathrm{R}^{3 \mathrm{~N}}$, and $\hat{T}$ is the correlation tensor given by

$$
T_{i_{1} \ldots i_{N}} \equiv E\left(\vec{x}_{1}^{\left(i_{1}\right)}, \vec{x}_{2}^{\left(i_{2}\right)}, \ldots, \vec{x}_{N}^{\left(i_{N}\right)}\right),
$$

where $\vec{x}_{j}^{\left(i_{j}\right)}$ is a unit directional vector of the local coordinate system of the $j$ th observer; $i_{j}=1,2,3$ gives the full set of orthogonal vectors defining the local Cartesian coordinates. Obviously the assumed form of (2.2) implies rotational invariance, because the correlation function does not depend on the coordinate systems used by the observers. Rotational invariance simply states that the value of $E\left(\vec{n}_{1}, \vec{n}_{2}, \ldots, \vec{n}_{N}\right)$ cannot depend on the local coordinate systems used by the $N$ observers.

Assume that one knows the values of all $3^{N}$ components of the correlation tensor, $T_{i_{1} \ldots i_{N}}$, which are obtainable by performing specific $3^{N}$ measurements of the correlation function, (cf. Eq. (2.3)). Then, with the use of the formula (2.2) we can reproduce the values of the correlation functions for all other possible sets of local settings. Using this rotationally invariant structure of the correlation function, we shall derive a necessary condition for the existence of a local realistic theory of the experimental correlation function given in (2.2). If the correlation 
function is described by the local realistic theory, then the correlation function must be simulated by the following structure

$$
E_{\mathrm{LR}}\left(\vec{n}_{1}, \vec{n}_{2}, \ldots, \vec{n}_{N}\right)=\int d \lambda \rho(\lambda) I^{(1)}\left(\vec{n}_{1}, \lambda\right) I^{(2)}\left(\vec{n}_{2}, \lambda\right) \cdots I^{(N)}\left(\vec{n}_{N}, \lambda\right),
$$

where $\lambda$ is some local hidden variable, $\rho(\lambda)$ is a probabilistic distribution, and $I^{(j)}\left(\vec{n}_{j}, \lambda\right)$ is the predetermined "hidden" result of the measurement of the dichotomic observable $\vec{n} \cdot \sigma$ with values \pm 1 .

Let us parametrize the arbitrary unit vector in the plane defined $\vec{x}_{j}^{(1)}$ and $\vec{x}_{j}^{(2)}$ in the following way:

$$
\vec{n}_{j}\left(\alpha_{j}\right)=\cos \alpha_{j} \vec{x}_{j}^{(1)}+\sin \alpha_{j} \vec{x}_{j}^{(2)} .
$$

We show that scalar product of any local realistic correlation function

$$
E_{\mathrm{LR}}\left(\alpha_{1}, \alpha_{2}, \ldots, \alpha_{N}\right)=\int d \lambda \rho(\lambda) I^{(1)}\left(\alpha_{1}, \lambda\right) I^{(2)}\left(\alpha_{2}, \lambda\right) \cdots I^{(N)}\left(\alpha_{N}, \lambda\right),
$$

with the rotationally invariant correlation function, that is,

$$
E\left(\alpha_{1}, \alpha_{2}, \ldots, \alpha_{N}\right)=\hat{T} \cdot\left(\vec{n}_{1}\left(\alpha_{1}\right) \otimes \vec{n}_{2}\left(\alpha_{2}\right) \otimes \cdots \otimes \vec{n}_{N}\left(\alpha_{N}\right)\right)
$$

is bounded by a specific number dependent on $\hat{T}$. We define the scalar product $\left(E_{\mathrm{LR}}, E\right)$ as follows: We see that the maximal possible value of $\left(E_{\mathrm{LR}}, E\right)$ is bounded as:

$$
\left(E_{\mathrm{LR}}, E\right)=\int_{0}^{2 \pi} d \alpha_{1} \int_{0}^{2 \pi} d \alpha_{2} \ldots \int_{0}^{2 \pi} d \alpha_{N} E_{\mathrm{LR}}\left(\alpha_{1}, \alpha_{2}, \ldots, \alpha_{N}\right) E\left(\alpha_{1}, \alpha_{2}, \ldots, \alpha_{N}\right) \leq 4^{N} T_{\max },
$$

where $T_{\max }$ is the maximal possible value of the correlation tensor component, i.e.,

$$
T_{\max }=\max _{\beta_{1}, \beta_{2}, \ldots, \beta_{N}} E\left(\beta_{1}, \beta_{2}, \ldots, \beta_{N}\right),
$$

where $\beta_{j}$ is some angle.

A necessary condition for the existence of a local realistic description $E_{\mathrm{LR}}$ of the experimental correlation function

$$
E\left(\alpha_{1}, \alpha_{2}, \ldots, \alpha_{N}\right)=E\left(\vec{n}_{1}\left(\alpha_{1}\right), \vec{n}_{2}\left(\alpha_{2}\right), \ldots, \vec{n}_{N}\left(\alpha_{N}\right)\right)
$$

that is, for $E_{\mathrm{LR}}$ to be equal to $E$, is that one has $\left(E_{\mathrm{LR}}, E\right)=(E, E)$. This implies the possibility of modeling $E$ by the local realistic correlation function $E_{\mathrm{LR}}$ given in (2.6). If we have $\left(E_{\mathrm{LR}}, E\right)<(E, E)$, then the experimental correlation function cannot be explainable by the local realistic theory.

In what follows, we derive the upper bound (2.8). Since the local realistic model is an average over $\lambda$, it is enough to find the bound of the following expression:

$$
\int_{0}^{2 \pi} d \alpha_{1} \cdots \int_{0}^{2 \pi} d \alpha_{N} I^{(1)}\left(\alpha_{1}, \lambda\right) \cdots I^{(N)}\left(\alpha_{N}, \lambda\right) \sum_{i_{1}, i_{2}, \ldots, i_{N}=1,2} T_{i_{1} i_{2} \ldots i_{N}} c_{1}^{i_{1}} c_{2}^{i_{2}} \cdots c_{N}^{i_{N}},
$$

where

$$
\left(c_{j}^{1}, c_{j}^{2}\right)=\left(\cos \alpha_{j}, \sin \alpha_{j}\right)
$$

and

$$
T_{i_{1} i_{2} \ldots i_{N}}=\hat{T} \cdot\left(\vec{x}_{1}^{\left(i_{1}\right)} \otimes \vec{x}_{2}^{\left(i_{2}\right)} \otimes \cdots \otimes \vec{x}_{N}^{\left(i_{N}\right)}\right),
$$


compare (2.2) and (2.3).

Let us analyze the structure of the integral (2.11). Note that (2.11) is a sum, with coefficients given by $T_{i_{1} i_{2} \ldots i_{N}}$, which is a product of the following integrals:

$$
\int_{0}^{2 \pi} d \alpha_{j} I^{(j)}\left(\alpha_{j}, \lambda\right) \cos \alpha_{j}
$$

and

$$
\int_{0}^{2 \pi} d \alpha_{j} I^{(j)}\left(\alpha_{j}, \lambda\right) \sin \alpha_{j}
$$

We deal here with integrals or, rather scalar products of $I^{(j)}\left(\alpha_{j}, \lambda\right)$ with two-orthogonal functions. One has

$$
\int_{0}^{2 \pi} d \alpha_{j} \cos \alpha_{j} \sin \alpha_{j}=0
$$

The normalized functions $\frac{1}{\sqrt{\pi}} \cos \alpha_{j}$ and $\frac{1}{\sqrt{\pi}} \sin \alpha_{j}$ form a basis of a real two-dimensional functional space, which we call $S^{(2)}$. Note further that any function in $S^{(2)}$ is of the form:

$$
A \frac{1}{\sqrt{\pi}} \cos \alpha_{j}+B \frac{1}{\sqrt{\pi}} \sin \alpha_{j}
$$

where $A$ and $B$ are constants, and that any normalized function in $S^{(2)}$ is given by

$$
\cos \psi \frac{1}{\sqrt{\pi}} \cos \alpha_{j}+\sin \psi \frac{1}{\sqrt{\pi}} \sin \alpha_{j}=\frac{1}{\sqrt{\pi}} \cos \left(\alpha_{j}-\psi\right) .
$$

The norm $\left\|I^{(j) \|}\right\|$ of the projection of $I^{(j)}$ into the space $S^{(2)}$ is given by the maximal possible value of the scalar product $I^{(j)}$ with any normalized function belonging to $S^{(2)}$, that is,

$$
\left\|I^{(j) \|}\right\|=\max _{\psi} \int_{0}^{2 \pi} d \alpha_{j} I^{(j)}\left(\alpha_{j}, \lambda\right) \frac{1}{\sqrt{\pi}} \cos \left(\alpha_{j}-\psi\right) .
$$

Because $\left|I^{(j)}\left(\alpha_{j}, \lambda\right)\right|=1$, one has $\left\|I^{(j) \|}\right\| \leq 4 / \sqrt{\pi}$.

Since $\frac{1}{\sqrt{\pi}} \cos \alpha_{j}$ and $\frac{1}{\sqrt{\pi}} \sin \alpha_{j}$ are two-orthogonal basis functions in $S^{(2)}$, one has

$$
\int_{0}^{2 \pi} d \alpha_{j} I^{(j)}\left(\alpha_{j}, \lambda\right) \frac{1}{\sqrt{\pi}} \cos \alpha_{j}=\cos \beta_{j}\left\|I^{(j) \|}\right\|
$$

and

$$
\int_{0}^{2 \pi} d \alpha_{j} I^{(j)}\left(\alpha_{j}, \lambda\right) \frac{1}{\sqrt{\pi}} \sin \alpha_{j}=\sin \beta_{j}\left\|I^{(j) \|}\right\|,
$$

where $\beta_{j}$ is some angle. Using this fact, one can put the value of (2.11) into the following form:

$$
(\sqrt{\pi})^{N} \prod_{j=1}^{N}\left\|I^{(j) \|}\right\| \times \sum_{i_{1}, i_{2}, \ldots, i_{N}=1,2} T_{i_{1} i_{2} \ldots i_{N}} d_{1}^{i_{1}} d_{2}^{i_{2}} \cdots d_{N}^{i_{N}}
$$

where

$$
\left(d_{j}^{1}, d_{j}^{2}\right)=\left(\cos \beta_{j}, \sin \beta_{j}\right) .
$$

Let us look at the expression

$$
\sum_{i_{1}, i_{2}, \ldots, i_{N}=1,2} T_{i_{1} i_{2} \ldots i_{N}} d_{1}^{i_{1}} d_{2}^{i_{2}} \cdots d_{N}^{i_{N}} .
$$


Formula (2.23) shows that we deal here with two-dimensional unit vectors $\vec{d}_{j}=\left(d_{j}^{1}, d_{j}^{2}\right), j=1,2, \ldots, N$; therefore (2.24) is equal to $\hat{T} \cdot\left(\vec{d}_{1} \otimes \vec{d}_{2} \otimes \cdots \otimes \vec{d}_{N}\right)$. That is, it is a component of the tensor $\hat{T}$ in the directions specified by the vectors $\vec{d}_{j}$. If one knows all the values of $T_{i_{1} i_{2} \ldots i_{N}}$, one can always find the maximal possible value of such a component, and it is equal to $T_{\max }$ of Eq. (2.9).

Therefore, since $\left\|I^{(j) \|}\right\| \leq 4 / \sqrt{\pi}$ the maximal value of (2.22) is $4^{N} T_{\max }$, and finally one has

$$
\left(E_{\mathrm{LR}}, E\right) \leq 4^{N} T_{\max }
$$

Please note that relation (2.25) is a generalized Bell inequality. A local realistic models must satisfy it. Below we show that if one replaces $E_{\mathrm{LR}}$ by $E$ one may have a violation of the inequality (2.25). One has

$$
\begin{aligned}
(E, E) & =\int_{0}^{2 \pi} d \alpha_{1} \int_{0}^{2 \pi} d \alpha_{2} \cdots \int_{0}^{2 \pi} d \alpha_{N}\left(\sum_{i_{1}, i_{2}, \ldots, i_{N}=1,2} T_{i_{1} i_{2} \ldots i_{N}} c_{1}^{i_{1}} c_{2}^{i_{2}} \cdots c_{N}^{i_{N}}\right)^{2} \\
& =\pi^{N} \sum_{i_{1}, i_{2}, \ldots, i_{N}=1,2} T_{i_{1} i_{2} \ldots i_{N}}^{2} .
\end{aligned}
$$

Here, we use the fact that $\int_{0}^{2 \pi} d \alpha_{j} c_{j}^{\alpha} c_{j}^{\beta}=\pi \delta_{\alpha, \beta}$, because $c_{j}^{1}=\cos \alpha_{j}$ and $c_{j}^{2}=\sin \alpha_{j}$.

The structure of condition (2.25) and the value (2.26) suggests that the value of (2.26) does not have to be smaller than (2.25). That is there may be such correlation functions $E$, which have the property that for any $E_{\mathrm{LR}}$ one has $\left(E_{\mathrm{LR}}, E\right)<(E, E)$, which implies the impossibility of modeling $E$ by the local realistic correlation function $E_{\mathrm{LR}}$.

We shall present an important quantum state. Consider the following $N$-qubit Greenberger-Horne-Zeilinger (GHZ) state [13]

$$
|\psi\rangle=\frac{1}{\sqrt{2}}\left(|z+\rangle_{1} \cdots|z+\rangle_{N}+|z-\rangle_{1} \cdots|z-\rangle_{N}\right),
$$

where $|z \pm\rangle_{j}$ is the eigenstate of the local $\sigma_{z}$ operator of the $j$ th observer. We introduce a mixture of GreenbergerHorne-Zeilinger correlations and white noise:

$$
\rho=V|\psi\rangle\langle\psi|+(1-V) \rho_{\text {noise }},
$$

where $|\psi\rangle$ is the GHZ state and $\rho_{\text {noise }}=\frac{1}{2^{N}} I$ is the random noise admixture. The value of $V$ can be interpreted as the reduction factor of the interferometric contrast observed in the $N$-particle correlation experiment.

Imagine $N$ observers who can choose between two-orthogonal directions of spin measurement, $\vec{x}_{j}^{(1)}$ and $\vec{x}_{j}^{(2)}$ for the $j$ th one. Let us assume that the source of $N$ entangled spin-carrying particles emits them in a state, which can be described as noisy Greenberger-Horne-Zeilinger correlations, given in (2.28). We can show that if the observers limit their settings to $\vec{x}_{j}^{(1)}=\hat{x}_{j}$ and $\vec{x}_{j}^{(2)}=\hat{y}_{j}$ there are

$$
2^{N}-1
$$

components of $\hat{T}$ of the value $\pm V$. These are $T_{11 \ldots 1}$ and all components that except from indices 1 have an even number of indices 2 . Other $x-y$ components vanish. 
It is easy to see that $T_{\max }=V$ and $\sum_{i_{1}, i_{2}, \ldots, i_{N}=1,2} T_{i_{1} i_{2} \ldots i_{N}}^{2}=V^{2} 2^{N-1}$. Then we have $\left(E_{\mathrm{LR}}, E\right) \leq 4^{N} V$ and $(E, E)=\pi^{N} V^{2} 2^{N-1}$. For $N \geq 4$ and $V$ given by

$$
2\left(\frac{2}{\pi}\right)^{N}<V \leq \frac{1}{\sqrt{2^{N-1}}}
$$

we see the fact that there exist two-setting local realistic models for the infinite measurement directions $\left(A_{1}, A_{2}\right),\left(A_{2}, A_{3}\right),\left(A_{3}, A_{4}\right), \ldots$ in consideration and these models $\left(A_{1}, A_{2}\right),\left(A_{2}, A_{3}\right),\left(A_{3}, A_{4}\right), \ldots$ construct infinitesetting local realistic models $\left(A_{1}, A_{2}, A_{3}, A_{4}, \ldots\right)$ because we suppose they are non-contextual local realistic models. And we have a violation of the generalized Bell inequality:

$$
\left(E_{L R}, E\right)<(E, E) .
$$

That is, non-contextual local realistic models violate the generalized Bell inequality.

As it is shown in [14] if the correlation tensor satisfies the following condition:

$$
\sum_{i_{1}, i_{2}, \ldots, i_{N}=1,2} T_{i_{1} i_{2} \ldots i_{N}}^{2} \leq 1
$$

then there always exists two-setting local realistic model for the set of correlation function values for all directions lying in a plane. For our example the condition (2.32) is met whenever $V \leq \frac{1}{\sqrt{2^{N-1}}}$. Nevertheless we have a violation of the generalized Bell inequality for $V>2\left(\frac{2}{\pi}\right)^{N}$.

The situation is such that for $V \leq \frac{1}{\sqrt{2^{N-1}}}$ for all two-settings-per-observer experiments we can construct a local realistic theory for the values of the correlation function for the settings chosen in the experiment. These theories must be consistent with each other if we want to extend their validity beyond the $2^{N}$ settings to which each of them pertains.

Here we suppose they are non-contextual local realistic models. Then we can extend their validity beyond the $2^{N}$ settings to which each of them pertains. Our calculations clearly indicate that this is possible for $V>2\left(\frac{2}{\pi}\right)^{N}$. And we have the violation of the generalized Bell inequality for $V>2\left(\frac{2}{\pi}\right)^{N}$. Therefore, we discuss incompleteness in the Bell theorem by using non-contextual local realistic models.

In the contextual scenario, there must be the essential difference between infinite-setting model and two-setting model. This says that we must distinguish infinite-setting model from two-setting model. And we can classify local realistic theories in this case.

\section{Conclusions and Discussions}

In conclusions, in the non-contextual scenario, there has not been the essential difference between infinitesetting model and two-setting model. And we cannot have classified local realistic theories in this case. This has said that we can construct infinite-setting model from two-setting model. Surprisingly we can have discussed incompleteness in the Bell theorem by using non-contextual models. On the other hand, in the contextual scenario, there has been the essential difference between infinite-setting model and two-setting model. This has said that we must distinguish infinite-setting model from two-setting model. And we can have classified local realistic theories in this case. 


\section{Note}

On behalf of all authors, the corresponding author states that there is no conflict of interest.

\section{REFERENCES}

[1] A. Einstein, B. Podolsky, N. Rosen, Can Quantum-Mechanical Description of Physical Reality Be Considered Complete?, Phys. Rev. 47 (1935) 777-780.

[2] J. S. Bell, On the Einstein Podolsky Rosen Paradox, Physics, 1 (3) (1964), 195-200.

[3] A. Aspect, P. Grangier, G. Roger, Experimental Tests of Realistic Local Theories via Bell's Theorem, Phys. Rev. Lett. 47 (1981), $460-463$.

[4] A. Aspect, P. Grangier, G. Roger, Experimental Realization of Einstein-Podolsky-Rosen-Bohm Gedankenexperiment: A New Violation of Bell's Inequalities, Phys. Rev. Lett. 49 (1982), 91-94.

[5] A. Aspect, J. Dalibard, G. Roger, Experimental Test of Bell's Inequalities Using Time- Varying Analyzers, Phys. Rev. Lett. 49 (1982), 1804-1807.

[6] J.F. Clauser, M.A. Horne, Experimental consequences of objective local theories, Phys. Rev. D. 10 (1974), 526-535.

[7] E.P. Wigner, On Hidden Variables and Quantum Mechanical Probabilities, Amer. J. Phys. 38 (1970), 1005-1009.

[8] C. Raman, M. Kohl, R. Onofrio, D.S. Durfee, C.E. Kuklewicz, Z. Hadzibabic, W. Ketterle, Evidence for a Critical Velocity in a Bose-Einstein Condensed Gas, Phys. Rev. Lett. 83 (1999), 2502-2505.

[9] H. Geurdes, K. Nagata, T. Nakamura, A. Farouk, A note on the possibility of incomplete theory, ArXiv:1704.00005 [Physics]. (2019).

[10] K. Nagata, T. Nakamura, and H. Geurdes, Incompleteness in the Bell Theorem Using Non-contextual Local Realistic Model, Int. J. Theor. Phys. 59 (2020), 313-320.

[11] K. Nagata, W. Laskowski, M. Wieśniak, M. Żukowski, RotationalInvariance as an Additional Constraint on Local Realism, Phys. Rev. Lett. 93 (2004), 230403.

[12] K. Nagata, J. Phys. A: Math. Theor. 41, 155308 (2008).

[13] D. M. Greenberger, M. A. Horne, and A. Zeilinger, Going Beyond Bell's Theorem, in Bell's Theorem, Quantum Theory and Conceptions of the Universe, edited by M. Kafatos (Kluwer Academic, Dordrecht, The Netherlands, 1989), p. 69.

[14] M. Żukowski, Č. Brukner, Bell's Theorem for General N -Qubit States, Phys. Rev. Lett. 88 (2002), 210401. 